\title{
LA FILOSOFÍA DE LA TECNOLOGÍA EN LA FORMACIÓN DE INGENIEROS: ALGUNAS IDEAS SOBRE LA EXPERIENCIA DE CUBA
}

\author{
LAMAR, Adolfo Ramos (Brasil, Santa Catarina, Blumenau) ${ }^{1 *}$ \\ ROACH, Eduardo Francisco Freyre (Cuba, Mayabeque, San José de las Lajas) ${ }^{2 * \star}$ \\ ${ }^{1}$ Universidade Regional de Blumenau \\ 'Universidad Agraria de la Habana \\ ORCID ID: https://orcid.org/0000-0003-1164-1172* \\ ORCID ID: https://orcid.org/0000-0002-2267-1564*
}

\begin{abstract}
RESUMEN
La tecnología vertiginosamente avanza e impacta en el modo de encarar la relación entre el ser humano y el mundo, el sentido de nuestras vidas, el conocimiento de la realidad y la gobernabilidad social. De ahí que, como nunca antes, la tecnología haya devenido en un tema que no solo se plantea en las diferentes ramas de la filosofía, sino también que suscita un tratamiento especializado en los confines de lo que se conoce como Filosofía de la Tecnología. Esta materia debería ser tomada en serio por quienes tienen que ver con el desarrollo tecnológico y sus aplicaciones, en especial los ingenieros y quienes intervienen en su formación y capacitación. Este trabajo aborda la introducción de temas de Filosofía de la Tecnología en los cursos de Ingenierías en Cuba, teniendo en cuenta su demanda social, fundamento epistemológico, así como los escenarios y estrategias de su enseñanza y aprendizaje. Como base empírica, se utilizaron los currículos y planes de estudios de carreras ingenieriles y se realizó una sistematización de las experiencias docentes de los autores como profesores en cursos de Ingenierías. Se concluye que la experiencia cubana en esta materia está relacionada con su contexto educacional y social.
\end{abstract}

\section{PALABRAS CLAVE}

Filosofía. Tecnología. Epistemología. Formación. Ingenierías. Cuba.

\section{A FILOSOFIA DA TECNOLOGIA NA FORMAÇÃO DE ENGENHEIROS: ALGUMAS IDEIAS SOBRE A EXPERIÊNCIA DE CUBA}

\section{RESUMO}

A tecnologia está avançando rapidamente e impacta na maneira de abordar a relação entre o ser humano e o mundo, o significado de nossas vidas, o conhecimento da realidade e a governabilidade social. Assim, a tecnologia tornou-se um assunto que não apenas surge nos diferentes ramos da filosofia, mas suscita ainda um tratamento especializado nos limites do que é conhecido como Filosofia da Tecnologia. Esse assunto deve ser levado a sério por aqueles que têm a ver com o desenvolvimento tecnológico e suas aplicações, especialmente engenheiros e aqueles envolvidos em sua formação e educação. Este trabalho aborda a introdução de temas de Filosofia da Tecnologia nos cursos de Engenharia em Cuba, considerando sua demanda social e base epistemológica, assim como os cenários e estratégias de seu ensino e aprendizagem. Como base empírica, foram utilizados os currículos e planos de carreiras de Engenharia e realizou-se uma sistematização das experiências docentes dos autores como professores em cursos de Engenharia. Conclui-se que a experiência cubana nesta questão está relacionada ao seu contexto educacional e social.

\section{PALAVRAS-CHAVE}

Filosofia. Tecnologia. Epistemologia. Formação. Engenharia. Cuba.

Educação \& Formação, Fortaleza, v. 4, n. 12, p. 3-20 set./out. 2019

DOI: https://doi.org/10.25053/redufor.v4i12.1425

http://seer.uece.br/redufor 


\title{
THE PHILOSOPHY OF TECHNOLOGY IN THE TRAINING OF ENGINEERS:
}

\section{SOME IDEAS ABOUT THE EXPERIENCE OF CUBA}

\begin{abstract}
The technology is rapidly advancing and impacts on the way of approaching the relationship between the human being and the world, the meaning of our lives, the knowledge of reality, and social governance. Hence, as never before, technology has become a subject that not only arises in the different branches of philosophy, but also that a specialized treatment in the confines of what is known as Philosophy of Technology. This matter should be taken seriously by those who have to do with technological development and its applications, especially engineers, and those involved in their training and education. This paper deals with the introduction of Philosophy of Technology topics in the courses of Engineering in Cuba, considering their social demand, epistemological foundation, as well as the scenarios and strategies of their teaching and learning. As an empirical basis, the curricula and syllabuses of engineering careers were used, and a systematization of the teaching experiences of the authors as professors in engineering courses was carried out. We conclude that the Cuban experience in this matter is related to its educational and social context.
\end{abstract}

\section{KEYWORDS}

Philosophy. Technology. Epistemology. Training. Engineering Cuba.

\section{INTRODUCCIÓN}

Aunque desde la Antigüedad se viene filosofando sobre la técnica (MITCHAM, 1989), existe amplio consenso en que, desde finales del XIX hasta nuestros días, lo tecnológico viene cobrando más y más auge en nuestras vidas. E. Kapp (1808-1896) es considerado el fundador de la Filosofía de la Técnica con su libro Grundlinien einer Philosophie der Technik (Fundamentos de una filosofía de la técnica), publicado en 1877. Otros importantes estudiosos fueron F. Dessauer, P. K. Engelmeier, L. Mumford, J. Ortega y Gasset y M. Heidegger. Moran Seminario (2006) destaca el papel de la Verein Deustcher Ingenieure (Sociedad Alemana de Ingenieros), de algunas universidades politécnicas alemanas y del físico, radiólogo, filósofo y empresario alemán Friedrich Dessauer en el aumento de su importancia. Lamar e Roach (1987), preocupados por la formación filosófica de los ingenieros en América Latina y Caribe, colocan que el filósofo español Juan David García Bacca (1901-1992) tuvo una importante influencia en el pensamiento latinoamericano sobre la ciencia y la técnica. Bacca impartió clases en diversos países latinoamericanos.

Educação \& Formação, Fortaleza, v. 4, n. 12, p. 3-20 set./out. 2019

DOI: https://doi.org/10.25053/redufor.v4i12.1425

http://seer.uece.br/redufor 
Lo cierto es que la época contemporánea trae consigo un filosofar sobre la tecnología de un calibre mayor. A decir de un destacado filósofo de la tecnología:

\begin{abstract}
Agora nós nos movemos para além da utilidade no sentido estrito da pergunta quanto ao tipo de mundo e ao modo de vida que emerge em uma sociedade moderna. Na medida em que tal sociedade tem base tecnológica, os problemas que surgem nesse questionamento referem-se ao campo da Filosofia da Tecnologia. Nós precisamos nos entender hoje no meio da tecnologia e o conhecimento propriamente técnico não pode nos ajudar. A Filosofia da Tecnologia pertence à autoconsciência de uma sociedade como a nossa. Nos ensina a refletir sobre o que tomamos como certo, especificamente a modernidade racional. A importância dessa perspectiva não pode ser subestimada. (FEENBERG, 2010, p. 39-40).
\end{abstract}

Sin embargo, la tecnología como objeto de reflexión filosófica es de crucial importancia no solo para los profesionales de la filosofía, sino también para todos que de una u otra forma tienen que ver con el desarrollo tecnológico, su transferencia, introducción y difusión. Destaquemos aquí a los ingenieros y quienes se encargan de su formación, capacitación y empleo.

Concordamos en que la Filosofía de la Tecnología es necesaria para los ingenieros, pues les ayuda a defenderse de las críticas filosóficas y tomar decisiones adecuadas en el ejercicio de su profesión. Pues la filosofía les puede ayudar a entender la ingeniería misma y su filosófico (MITCHAM, 1998). Es por ello que con gran fuerza se viene reclamando la necesidad de que los conocimientos de la Filosofía de la Tecnología tengan mayor presencia en la formación de técnicos ingenieros.

La creciente y acelerada tecnificación o ingenierización de la sociedad se acompaña de sueños, pero también de pesadillas, a ser evitadas por nuevas generaciones de ingenieros, dotados de compromisos social, ético y humanista, y sobre todo no siendo cómplices de decisiones tecnocráticas en la sociedad, es decir, basadas solo en la eficiencia y eficacia tecnológicas. En la opinión de Oliveira (2001, p. 111), "[...] as teorias destinadas a servir as necessidades da engenharia não podem ser, como sucedia no passado, de carácter exclusivamente quantitativo, nem mesmo exclusivamente lógico-matemático, ou físico". Ya para Gatti (2004, p. 13): "Em si, tabelas, indicadores, testes de significância, etc., nada dizem. O significado dos resultados é dado pelo pesquisador em função de seu estofo teórico".

Educação \& Formação, Fortaleza, v. 4, n. 12, p. 3-20 set./out. 2019 
No debe perderse de vista que tal imperativo transciende el campo de las carreras tecnológicas e ingenieriles (KOERICH et al., 2006). La ciencia también encontró obstáculos filosóficos en su inicio (CÁRDENAS BARRETO, 2015): Así, es importante mencionar a G. Bachelard $(1996,1997)$ con sus conceptos de "vigilância epistemológica" y "obstáculos epistemológicos".

Debemos tener cuidado al minimizar la relación de la filosofía para la medicina veterinaria, ingeniería y medicina. Vivimos tiempos en Cuba en que parecido enfoque conlleva a minimizar la formación de especialistas en filosofía y ciencias sociales y también de los contenidos de estas materias en el currículo de la formación de ingenieros. Hay que comprender también que el interés por el estudio de la historia de ciencias y de las tecnologías tiene su condicionamento en el hecho de que ambas han experimentado considerables cambios revolucionarios y, por otra parte, los cambios en la ciencia y la tecnología afectan mucho a la cultura y a la propia filosofía. Eso quiere decir que es necesario saber cómo se producen allí las revoluciones científicas (KUHN, 1997).

Existen evidencias contundentes de que el bienestar social está puntualmente relacionado con la buena gobernación, es decir, la toma atinada de decisiones, la cual, a su vez, depende "[...] de ciências humanas como ciência social e filosofia, porque são estas disciplinas que fazem esse tipo de trabalho" (BARIFOUSE, 2019, p. 1). En Cuba, se viene tomando consciencia de esta problemática, la cual se refleja en el modo en que se encara hoy el aprendizaje de contenidos de filosofía de la tecnología, ya no solo en las carreras de perfil tecnológico-ingenieril, sino también de ciencias humanas y sociales.

Dada la situación problemática esbozada, el presente trabajo tiene como objetivo central abordar el tratamiento que en los últimos 20 años se le viene otorgando a los contenidos de Filosofía de la Tecnología en las carreras de ingenierías en Cuba. Se ha considerado aquí, en primer lugar, la demanda social tanto en el contexto global como local (nacional), que viene condicionado a la necesidad de la presencia de esos contenidos en el currículo para la formación y capacitación de ingenieros. En segundo lugar, se exponen los fundamentos epistemológicos que pautan la docencia y el aprendizaje de esos contenidos: el enfoque teórico directriz que se viene adoptando para concebir e impartir esos contenidos. Por último, es decir, en tercer lugar, se caracterizan los escenarios de organización y estrategias de su enseñanza y aprendizaje.

Educação \& Formação, Fortaleza, v. 4, n. 12, p. 3-20 set./out. 2019 
Como base empírica documental, se utilizaron los currículos y planes de estudios de carreras ingenieriles y los programas y bibliografía básica de los cursos donde los contenidos de Filosofía de la Tecnología se tratan. Nos propusimos resumir nuestra experiencia docente, de investigación y de debate durante los 27 años que trabajamos como profesores e investigadores en cursos de ingeniería de Cuba, de Brasil y de otros países de América Latina.

\section{DEMANDA ACADÉMICA, SOCIAL, GLOBAL Y LOCAL DE LA FILOSOFÍA DE LA TECNOLOGÍA, SU DOCENCIA Y APRENDIZAJE EN LAS CARRERAS DE INGENIERÍA}

A partir de los años 1990, la Filosofía de la Tecnología se desdobla en dos tradiciones: una toma como referente a Ernst Kapp y su idea de una Filosofía de la Tecnología ligada a la ingeniería; y la otra tiene que ver con los trabajos de Lewis Mumford, Ortega y Gasset, Martín Heidegger, Jacques Ellul, Paul Durbin, Don Ihde y Larry Hickman, de tradición humanista, que coloca a la tecnología en la perspectiva de reflexión de las ciencias humanas (MITCHAM, 2001). Ambas tradiciones vienen desarrollándose en un contexto que precisamente demanda reflexión filosófica diferenciada sobre la tecnología.

Dado al grado en que la tecnología de hoy tiene presencia e impacto en nuestras vidas, obliga a replantear cuestiones filosóficas ancestrales de un modo diferente. Por ejemplo, tomemos la cuestión ¿Qué es el ser?, tema objeto de la rama de la filosofía denominada de ontología. Hoy, para abordar esta pregunta, debe considerarse que la categoría de ser contempla ya no solo la naturaleza, la mente y la sociedad, sino también la realidad creada por el ser humano, los productos artificiales y tecnológicos, en fin, el mundo de los artefactos y las decisiones basados en conocimientos técnicos, que van colonizando el ambiente en que se desenvuelven nuestras vidas. No es un secreto que, a diferencia de épocas anteriores, vivimos más y más en un mundo de tecnologías. Con solo mirar hasta donde el ser humano ha llegado con la informática, la biotecnología, la robótica, la nanotecnología, etc., alcanza para entender que ya no vivimos en un mundo en que la cuestión del ser apunta a definirlo como algo que existe independientemente de la voluntad de los seres humanos. Resumiendo:

Educação \& Formação, Fortaleza, v. 4, n. 12, p. 3-20 set./out. 2019 
O significado e os fins das coisas são algo que nós criamos e não algo que descobrimos. A brecha entre homem e mundo se alarga consequentemente. Nós não estamos em casa no mundo, nós conquistamos o mundo. Esta diferença está relacionada com a nossa ontologia básica. A pergunta que nós nos dirigimos ao ser não é o que é, mas como funciona. (FEENBERG, 2010, p. 42).

¿En antropología filosófica, podemos seguir discutiendo la cuestión de qué es el ser humano, cuál es su esencia y destinación cósmica, pasando por alto su comportamiento tecnológico y en qué medida transforma su vida, su historia y su medio ambiente? Por supuesto que no. La definición clásica del hombre como ser racional formulada por Aristóteles está siendo desafiada por el hecho de que nuestro comportamiento tecnológico y la dependencia de lo tecnológico pasan a primer plano, amenazando con limitar nuestra razón y naturaleza a fines tecnológicos. Un ejemplo son las innovaciones en ingeniería informática, que vienen convirtiéndose en algo esencial en vida cotidiana personal e institucional. Un caso extremo que viene a la mente es la adicción a la informática, particularmente la adicción a videojuegos online, ya reconocida por la Organización Mundial de la Salud (OMS) como un tipo de enfermedad mental. Y se teme, no sin razones, que la tecnología termine secuestrando la toma de decisiones en todos los ámbitos, sobre todo en lo que atañe a la salud, la estética corporal, las relaciones interpersonales y la gobernabilidad social.

La reflexión acerca de la demarcación e interrelación entre conocimiento científico y conocimiento tecnológico obliga a repensar el problema filosófico ancestral de la verdad de los conocimientos. La epistemología surge como rama de la filosofía, donde interesa la reflexión sobre el alcance del conocimiento científico, sus fundamentos ontológicos y gnoseológicos. Los presupuestos ontológicos se refieren a la naturaleza de la realidad en general y de la realidad social $u$ objeto de estudio. $Y$ los presupuestos gnoseológicos tratan de la idea de conocimiento científico. La educación es un ámbito de transmisión, generación, así como de interacción (conflictiva o amistosa) entre conocimientos, saberes y tecnologías.

Vemos el auge de la perspectiva sobre la tecnología no como mera ciencia aplicada y la Filosofía de la Tecnología no como subrama de la Filosofía de la Ciencia (MEDINA, 1995). En este sentido, al conocimiento tecnológico, a diferencia del conocimiento científico, suelen atribuirse características específicas, por ejemplo, se

Educação \& Formação, Fortaleza, v. 4, n. 12, p. 3-20 set./out. 2019 
habla de su carácter prescriptivo por cuanto con el conocimiento se busca factibilidad, confiabilidad, eficiencia, optimización, simulación, modelación y relación adecuada costo-beneficio. También suele hacerse referencia a su carácter proyectivo, normativo, funcional, instrumental, nomo-pragmático, posibilístico, analógico/visual, etc. "Debido a la especificidad del conocimiento tecnológico, algunos autores se inclinan a abandonar la tradicional definición del conocimiento como creencia verdadera justificada" (CUPANI, 2006, p. 358). Sin embargo, en esta materia de gnoseología o teoría del conocimiento, hoy se plantea el problema de que la pretensión del conocimiento verdadero y justificado racionalmente está cediéndole el terreno al conocimiento que solo tiene valor instrumental.

Es necesario advertir que, si bien la tecnología general se enfoca con relativa independencia de la ciencia, parece ser que la ingeniería, aunque en esa misma perspectiva, continúa viéndose como "[...] la aplicación práctica de principios científicos en una forma creativa" (ISNAD, 2018, p. 7). El problema que merece reflexión es si esta definición desconsidera que en el trabajo ingenieril intervienen múltiples saberes.

Por último, tengamos en mente que la tecnología actual suscita nuevos debates sobre nuestras nociones de la bondad y la justicia. En épocas anteriores pudo haber sido moralmente loable decir que un avance tecnológico demanda aplicación necesaria, pero hoy en día esta perspectiva no hace más que desplazar la ética a un papel secundario o subsidiario de la tecnología y, por si fuera poco, propone que la tecnología escape a su escrutinio. Como la tecnología actual suscita esperanzas, pero también dolores de cabeza, lógicamente, apologéticas o críticas, de tecnofobia y la tecnofilia, "ya con moderación, ya con fanatismo" (BUNGE, 2018, p. 2).

Este marco global de entendimiento de la importancia de la tecnología y de la Filosofía de la Tecnología naturalmente influye en la toma de consciencia en Cuba de la necesidad de la introducción de conocimientos de la Filosofía de la Tecnología en el currículo de las ingenierías en Cuba. Salta a la vista que, a partir de finales de 1980, los profesionales cubanos acceden por diversas vías a la bibliografía sobre el tema.

Si volteamos más y más la mirada al contexto local en esos tiempos, debemos detenernos en el hecho de que el gobierno intensifica su perspectiva de ver en la ciencia y la tecnología un factor importante para el desarrollo de la producción, los servicios y el bienestar de la sociedad. Pero no es menos cierto que durante esos años hay gran 
preocupación de que las tecnologías e innovaciones tecnológicas, en el mejor de los casos, tardaban en implementarse y, en el peor, quedaban sin aplicación práctica.

En Cuba, a partir de 1990, se establece un nuevo Sistema de Ciencia, Tecnología e Innovación Tecnológica (SCT+IT), para subvertir los sesgos asociados al modelo de Dirección Centralizada de la Ciencia y la Tecnología desde 1975. Con esta transformación el gobierno vislumbra también enfrentar la crisis económica que vino con el colapso de la Unión de Repúblicas Socialistas Soviéticas (URSS) y el socialismo y con el recrudecimiento del bloqueo que Estados Unidos impuso a Cuba en 1961.

Este SCT+IT busca mayor integración y sinergia entre el desarrollo científicotecnológico y el desarrollo económico-social. Al calor de este factor surgieron medidas como los polos científico-productivos y la constitución del Ministerio de Ciencia, Tecnología y Medio Ambiente (CITMA). Gran atención se le comenzó a otorgar a la informatización de la sociedad. Hoy en día, siguiendo la experiencia de la Universidad de la Informática, se proyectan la creación de otros parques científico-tecnológicos y la definición y creación de empresas de alta tecnología en otras universidades del país, que precisamente mejoren la relación universidad-sector productivo y social.

Como ocurre en una gran parte del mundo, en Cuba la problemática ambiental obliga a repensar las relaciones entre ciencia y tecnología, así como sus impactos. La relevancia de este aspecto se manifiesta desde la hora en punto en que la política científica del gobierno conecta esa problemática con la ciencia y la tecnología. Este es un factor que ha tenido gran repercusión y que le ha imprimido un vuelco a la gestión tecnológica en la agricultura y la investigación en ingeniería agronómica e ingeniería en mecanización agropecuaria. La concepción de la agricultura sostenible, como directriz tecnológica de la política agrícola oficial, está exigiendo cambios en el conocimiento ingenieril agropecuario.

En Cuba hay gran interés en la soberanía e independencia tecnológica, mensaje importante para profesionales ingenieros, en el sentido que indica la necesidad del diseño de sistemas ingenieriles basados en necesidades, condiciones y saberes endógenos (ALBERT PINO; CARMONA TAMAYO; ROMEO MATOS, 2018).

Por último, es necesario plantear que la gestión de la ciencia y la tecnología en Cuba viene poniendo énfasis en el incremento de los recursos humanos involucrados y la participación masiva en esta actividad. Se trata además de potenciar relaciones de 
diálogo y sinergia entre los autores sociales. Este imperativo demanda de los ingenieros mayor apertura hacia el trabajo multidisciplinario, colaborativo y cooperativo.

No es difícil darse cuenta de que en este contexto se demandan transformaciones en la formación de profesionales de la educación superior, ya no solo en cuanto a la necesidad de mejorar las interacciones entre producción, servicios, investigación y capacitación de profesionales, sino también en cuanto al enfoque de la ciencia, tecnología y sociedad.

\section{LOS FUNDAMENTOS EPISTEMOLÓGICOS DE LA ENSEÑANZA DE CONTENIDOS DE FILOSOFÍA DE LA TECNOLOGÍA PARA LOS INGENIEROS EN CUBA}

En Cuba el Marxismo-Leninismo, como filosofía e ideología oficial, continúa pautando la reflexión sobre la ciencia y la tecnología, aunque actualmente se vienen recepcionando otras perspectivas o enfoques. $Y$ esto se conecta también con los cambios en la política económica, científico-tecnológica y educacional.

Mitcham (1989, p. 89) plantea que el Marxismo soviético piensa la tecnología en los siguientes términos: "No hay nada que cuestionar a la tecnología, sino solo al contexto social en el que está inmersa [...]". De 1975 hasta finales de la década de 1980 este enfoque fue transferido en Cuba, lo cual concuerda con el acento tecnocrático de la política científica en esos años. No extraña que esa concepción llevó al exagerado peso que se le otorgó a la transferencia tecnológica linear o vertical desde el exterior hacia Cuba y también desde las instituciones científicas hacia los contextos productivos y de servicios.

En la circunstancia histórica de la crisis de 1990, cobró auge la consciencia de la necesidad del replanteamiento de la enseñanza del Marxismo-Leninismo en todas las carreras universitarias. Es la época en que con fuerza se habla de un MarxismoLeninismo no dogmático y en sinergia con el pensamiento del tercer mundo y de Cuba, así como la necesidad de tomar en cuenta otras perspectivas de enfoque de los problemas. Se abre paso también a la percepción de que la enseñanza del Marxismo debe dejar de ser abstracta y, en su lugar, a los perfiles profesionales o carreras.

De ahí entonces que se propició la recepción de diversos enfoques de la ciencia y la tecnología que precisamente desafían su imagen convencional, pautada sobre todo 
por la filosofía positivista. Uno de esos enfoques vino a Cuba incluso de la URSS y la RDA: los estudios de Cienciología o Teoría de la Ciencia, así como la relevancia que allí se le otorgó a los estudios de Historia de la Ciencia de John D. Bernal. A la par se observa la recepción de autores cubanos y latinoamericanos (Amílcar Herrera, Jorge Sábato, Osvaldo Sunkel, etc.) a las posiciones economicistas, tecnocráticodesarrollistas, que terminan abogando por la dependencia tecnológica de la región. Obras de autores norteamericanos y europeos, como Thomas S. Kuhn, Imre Lakatos, Paul K. Feyerabend, Robert K. Merton y otros, empezaron a tener gran consumo entre los profesionales cubanos. Se dio bienvenida y gran acogida a nuevos campos del saber, como la Sociología del Conocimiento y la Sociología de la Ciencia, los Social Studies of Science and Technology o los Estudios de Ciencia, Tecnología y Sociedad (CTS) (GONZÁLEZ GARCÍA; LÓPEZ CEREZO; LUJÁN, 1996; NÚÑEZ JOVER, 2001).

Todas esas fuentes contribuyeron a revalorizar la teoría marxista de la ciencia y la tecnología y la enseñanza de los contenidos correspondientes en las carreras universitarias, en especial en la carrera de ingeniería. En efecto, los programas de Marxismo comenzaron a transformarse en ese sentido, lo cual se manifiesta de forma más evidente y crucial en la introducción de la asignatura Problemas Sociales de la Ciencia y la Tecnología (PSCT) como cierre del curso o disciplina de Marxismo.

Anteriormente la estructura de la disciplina constaba de tres partes: La Filosofía del Marxismo (el Materialismo Dialéctico e Histórico), la Economía Política del Capitalismo y el Socialismo y el Socialismo Científico. Ahora el tratamiento de contenidos de Filosofía de la Tecnología, si bien aún continúa en esos encuadres, en los marcos de PSCT, trata de conectar la visión de la ciencia, la tecnología y la sociedad, pero no solo desde una óptica general, abstracta o descontextualizada, sino atendiendo, como se dijo, a los diferentes perfiles de carreras, facultades, universidades y entornos locales.

En los textos básicos para esta asignatura, en las carreras universitarias (DÍAZ CABALLERO et al., 2006; NÚÑEZ JOVER, 2001) suelen ser recurrentes contenidos que apuntan hacia la naturaleza de la ciencia y la tecnología, pero que van más allá de la pauta epistemológica del Marxismo sobre su entendimiento como fuerza productiva directa o componente de la superestructura institucional de la sociedad vista como Formación Económico-Social (FES). A decir de un destacado especialista cubano:

Educação \& Formação, Fortaleza, v. 4, n. 12, p. 3-20 set./out. 2019 
[...] la educación científica no puede apoyarse en imágenes caducas de la ciencia y la tecnología [...]. La discusión sobre los aspectos institucionales, sociales y culturales propios del trabajo científico contribuye a enriquecer las imágenes de la tecnociencia que los canales educativos, formales e informales suelen transmitir. (NÚÑEZ JOVER, 2001, p. 6).

Es decir, que los propósitos que se propone hoy en día la política científica y tecnológica en Cuba suponen desafiar esas imágenes caducas de la ciencia y la tecnología, que las presentan como actividades que, debido a su neutralidad, resultan por definición benefactores.

Los problemas del desarrollo científico y tecnológico vienen acompañados de esa imagen descontextualizada de la ciencia y la tecnología. Si se entiende la tecnología como mera ciencia aplicada, eso conlleva a muchas dificultades, como el desatender su naturaleza específica, la diversidad de criterios e intereses que hay que tomar en cuenta, la transferencia ofertista, linear y vertical de conocimientos, el excesivo poder de los expertos en ciencia y tecnología en la toma de decisiones, así como la invisibilización de sus riesgos y peligros. Ciertamente los PSCT desafían, o más bien cuestionan la perspectiva de justificar la transferencia tecnológica, linear, ofertista y vertical. Los contenidos de PSCT para ingenieros desarrollan la siguiente perspectiva:

\footnotetext{
El desarrollo científico-tecnológico del país demanda por necesidad un profundo proceso de transculturación, de acumulación de capacidades y actitudes para generar, incorporar, asimilar, adaptar, perfeccionar, copiar, apropiar y aplicar conocimientos y sus correspondientes tecnologías, en materia de ciencia, tecnología e innovación tecnológica. La ciencia nacional requiere ser pensada y explicada desde esa perspectiva, sin renunciar a nuestra identidad nacional, ni a los últimos avances de la ciencia universal. Todo puede ser asimilado de forma adecuada y sin extremos, con lo cual estaríamos enriqueciendo la cultura científica nacional con valiosos elementos del acervo científico y tecnológico mundial. (DÍAZ CABALLERO, 2016, p. 61).
}

Eso implica a decir del autor citado darle atención a la necesidad de sustituir la apuesta por las prácticas tecnológicas exógenas o foráneas, por una perspectiva transcultural, donde esas se asimilan y se integren creativamente con las endógenas. $\mathrm{Y}$, por otro lado, el ingeniero no debe perder de vista que muchas de las tecnologías actuales tienen un carácter elitista, en tanto se plantean temas tecnológicos:

[...] razonables solo para aquellos que pueden darse el lujo de acceder a estas cimas de la cultura científico-tecnológica actual y, algo irracional por

Educação \& Formação, Fortaleza, v. 4, n. 12, p. 3-20 set./out. 2019

DOI: https://doi.org/10.25053/redufor.v4i12.1425 
completo, para los cientos de millones de seres humanos que viven en la más espantosa pobreza, que no saben qué es un maestro, un médico y muchísimo menos un gen o una computadora; masas depauperadas para las cuales la frase 'no solo de pan vive el hombre' carece totalmente de sentido [...]. (DÍAZ CABALLERO; RUBIDO RODRÍGUEZ, 2011, p. 67).

Aunque ya existe gran debate sobre ese asunto, "[...] en la actualidad, son demasiado escasas las evidencias de componentes científicos y tecnológicos endógenos en las nuevas iniciativas de desarrollo e inversiones anunciadas" (MONTERO CABRERA, 2019, p. 6), pero, por lo menos en materia educativa, ya se ha consolidado ese requerimiento.

El entendimiento de la naturaleza social de tecnología, es decir, como proceso social y sus condicionamientos sociales, lleva a un segundo grupo de contenidos, que se refiere al impacto social de las tecnologías. Se espera que el profesional esté en condiciones de analizar la proyección, aplicación, difusión y transferencia de tecnologías, tomando en cuenta precisamente no solo sus condicionamientos e impactos sociales y ambientales. Eso coloca en primer orden la necesidad de capacitación del ingeniero en el trabajo y debate multidisciplinario sociohumanístico (DÍAZ CABALLERO, 2010).

Esa perspectiva implica la adopción de una actitud crítica que abre la posibilidad de discutir la absolutización de las aristas transformadoras, creativas, artificiales, así como la supuesta esencia dinámica y revolucionaria de por sí de las tecnologías. Por esa vía se toma consciencia de que las decisiones tecnológicas deben basarse no solo en el conocimiento científico que las fundamentan, su eficacia, o eficiencia, sino también en las condiciones sociales y los conocimientos de humanidades, filosofía y ciencias sociales.

La ingeniería se manifiesta "[...] como el elemento mediador o núcleo integrador, en el sentido técnico, de la ciencia, la tecnología y la técnica, o sea, el núcleo integrador de lo que se denomina tecnociencia o continuum científico-técnico" (DÍAZ CABALLERO; BORRERO, 2011, p. 4). Sin embargo, eso significa considerar su potencia de mediar con otros saberes. Por un lado, viene resultando anacrónica la idea de un ingeniero, desatendido de las contribuciones de las ciencias sociales, como un científico social, al cual el desarrollo de la ciencia, la tecnología y la ingeniería le resulte indiferente. Un ejemplo de ello es evidente cuando hoy en día el enfoque agroecológico en agronomía demanda el diálogo de los profesionales con el saber campesino, comunitario y local.

Educação \& Formação, Fortaleza, v. 4, n. 12, p. 3-20 set./out. 2019 
Digamos que a un ingeniero entrenado y con mayor olfato en la identificación y crítica de las condicionantes e impactos sociales de la ciencia y la tecnología le resultaría más fácil entender ese nivel de reflexión y practicarlo, es decir, proyectar su trabajo de investigación docente y de extensionismo con plena consciencia del contexto social, y no al margen de este. Eso implica mayor capacidad de escuchar, recepción y justo valorar la percepción, criterios y expectativas de otros actores sobre la necesidad y viabilidad de una determinada tecnología. Entre esos actores merece destaque, por un lado, el trabajo colaborativo de los ingenieros con los trabajadores, campesinos y otros actores sociales, pero, por otro lado, con los especialistas de otras ramas del saber, sea ingenieril, médico o de ciencias sociales y humanísticas.

Por último, un tercer bloque de contenidos de Filosofía de la Tecnología apunta hacia la responsabilidad social y ética de los ingenieros. El tratamiento de ese contenido en el currículo implica el debate de la cuestión de si la ingeniería por naturaleza es beneficiosa, perjudicial o neutral.

Del ingeniero cubano, a partir de los cursos de PSCT, se espera que comprenda los peligros a los que lleva la absolutización de una de las variables de esa ecuación. Cuando ese sesgo ocurre, se tiende a entender lo bueno y lo malo de la tecnología o de que algún diseño ingenieril radica solo en su calidad profesional, su aplicación, según las intenciones, y lo inesperado. Sin embargo, el enfoque que PSCT ofrece a esa problemática es que, a partir de que no se visualice solo el aspecto técnico o artefactual de una determinada propuesta tecnológica, se contemplen otras variables que van más allá, es decir, los condicionamientos sociales e impactos sociales. Pero lo más importante aquí es que la responsabilidad ingenieril, por lo planteado, no queda esclavizada por los estrechos criterios de eficiencia o eficacia técnica. O sea, esa responsabilidad no termina ahí ni limita a la contribución de un avance tecnológico al margen de otros criterios.

Por lo tanto, se exhorta a la formación de un ingeniero con plena consciencia de que su tributación social comienza desde la hora en punto en que proyecta un sistema, lo continúa a través del proceso de su confección y lo extiende hacia el contexto de su implementación, evaluación, monitoreo y seguimiento. Cuando el análisis de lo tecnológico se concentra en el producto y queda fuera el proceso, lógicamente se tenderá a ver que el compromiso del profesional se ve solo en el protagonismo creativo.

Educação \& Formação, Fortaleza, v. 4, n. 12, p. 3-20 set./out. 2019 


\section{ESCENARIOS Y ESTRATEGIAS DE LA ENSEÑANZA Y EL APRENDIZAJE DE CONOCIMIENTOS DE FILOSOFÍA DE LA TECNOLOGÍA EN LA FORMACIÓN DE INGENIEROS}

De hecho, la introducción de los PSCT resulta un escenario crucial para la enseñanza y el aprendizaje de las cuestiones de Filosofía de la Tecnología que conciernen al trabajo ingenieril. Como ya se dijo, esta materia se imparte en todas las carreras universitarias, incluso en las carreras de perfil humanístico. Atrás ha quedado el divorcio académico entre los profesionales de los diferentes perfiles. Este sería el escenario organizativo multidisciplinario que comparten todas las profesiones.

En segundo lugar, los contenidos de PSCT se extienden a la formación posgraduada. Para optar por categorías docentes y científicas, el profesional debe realizar un trabajo de PSCT conectado con su profesión, esferas de actuación profesional y entornos locales. Resulta muy importante que, en los programas de maestría y doctorado, suele aparecer el requisito de PSCT ya no solo en el requisito de examen, sino también en el aporte mismo de la investigación. $Y$, por otro lado, en las maestrías de PSCT no sorprende el acceso de profesionales de todos los perfiles profesionales. Como los contenidos de Filosofía de la Tecnología se abordan en pregrado y posgrado, eso permite no solo continuidad y desarrollo en la instrucción de esa materia, sino que mayor posibilidad de que las situaciones de la realidad científica y tecnológica mundial, nacional y local se conviertan en objeto de reflexión, estudio y debate en el contexto docente, y viceversa, es decir, que la producción de conocimiento en el espacio docente tenga aplicación en esa vida científica y tecnológica, tanto en la elaboración de una determinada política, programa, plan o proyecto de ciencia y tecnología, como de su aplicación y evaluación de sus resultados.

En tercer lugar, puede visualizarse la tendencia de los proyectos de investigación tecnológica e ingenieril en las instituciones universitarias a contemplar temáticas de PSCT y ya no es extraño que en esos proyectos participen profesionales tanto de la filosofía como de las ciencias sociales, sobre todo porque el ingeniero tiene que dejar bien en claro los condicionamientos e impactos sociales de su contribución. La presencia de esos contenidos en la formación de posgrado posibilita mayor posibilidad de que las

Educação \& Formação, Fortaleza, v. 4, n. 12, p. 3-20 set./out. 2019 
situaciones de la realidad científica y tecnológica mundial, nacional y local se conviertan en objeto de reflexión, estudio y debate en el contexto docente.

Por último, en Cuba, a pesar de la crisis económica, el Estado no ha disminuido su atención a la formación de profesionales, debido a su apuesta al desarrollo científicotecnológico como factor para superar esa crisis. No hay más que ver el proceso de ampliación de las universidades y su acceso en los entornos locales, provinciales y municipales. En esos entornos tampoco sorprende la existencia de un ingeniero que se desempeña como profesor de la sede universitaria local, con pleno conocimiento de ese entorno e incluso impartiendo los PSCT y los contenidos de Filosofía de la Tecnología.

Las estrategias docentes, por tal motivo, ya no quedan enclaustradas en lo que el profesor quiere enseñar, sino en lo que el estudiante aprende, de forma activa y participativa, en el contexto histórico-social en que realiza su responsabilidad y compromiso social. Es decir, que los problemas que se discuten en PSCT y otras disciplinas son situaciones reales del entorno del trabajo ingenieril.

\section{CONSIDERACIONES FINALES}

Si se está claro de que el desarrollo ingenieril no es más que la encarnación o consumación de una filosofía y que, al mismo tiempo, ese desarrollo plantea y replantea problemas filosóficos y pautas para el razonamiento filosófico, entonces no costará trabajo otorgarle relevancia a la filosofía en un currículo de ingeniería y la ingeniería en un currículo de filosofía, ni mucho menos el emprendimiento del reto pedagógico de transversalizar contenidos filosóficos en los cursos que tributan a la formación del profesional de la ingeniería.

En este sentido, la experiencia cubana en materia de introducción de contenidos de Filosofía de la Tecnología en el currículo de formación de ingenieros aporta algunas lecciones. Como hemos visto, en Cuba los contenidos de Filosofía de la Tecnología para los ingenieros se integran en la asignatura de Problemas Sociales de la Ciencia y la Tecnología, que responde a que el fenómeno tecnológico e ingenieril viene demandando su análisis desde múltiples aristas y también el trabajo mancomunado de profesionales de diversos perfiles. Es una materia que se imparte en todas las carreras y que resulta

Educação \& Formação, Fortaleza, v. 4, n. 12, p. 3-20 set./out. 2019 
un espacio de encuentro ya no solo entre profesionales, sino también entre actores sociales en los entornos sociales.

Un proyecto, por ejemplo, de ingeniería agronómica recaba de la participación no solo de ingenieros, matemáticos, físicos, biólogos e informáticos, sino también de sociólogos, historiadores, psicólogos, filósofos, campesinos y líderes de comunidades. Y, por otro lado, que se atiendan todos los diferentes aspectos que garantizan el éxito del proyecto en cuestión.

Ciertamente los PSCT abren en Cuba perspectivas importantes para repensar la formación de los ingenieros (GUTIÉRREZ; ACHÓN, 2018), sobre todo en la medida de que, en los marcos del aprendizaje de los contenidos de Filosofía de la Tecnología, el ingeniero amplía su cultura no solo en sentido estrecho profesional, sino también humanístico.

\section{REFERENCIAS}

ALBERT PINO, B.; CARMONA TAMAYO, E.; ROMEO MATOS, L. La ciencia en Cuba, una mirada a sus retos y proyecciones. Cubadebate. Disponible en: $<$ http://www.cubadebate.cu/especiales/2018/12/28/la-ciencia-en-cuba-una-mirada-a-susretos-y-proyecciones/\#.xqhsa1wzzpy>. Acceso el: 12 jun. 2019.

BACHELARD, G. A formação do espírito científico: contribuição para uma psicanálise do conhecimento. Rio de Janeiro: Contraponto, 1996.

BACHELARD, G. O racionalismo aplicado. Rio de Janeiro: Zahar, 1997.

BARIFOUSE R. Ciências humanas são tão importantes quanto exatas e biológicas, diz professora de Harvard. BBC News Brasil, São Paulo, 30 abr. 2019. Disponível em: <https://www.bbc.com/portuguese/brasil-48070180>. Acesso em: 12 jun. 2019.

BUNGE, M. Nada grandioso se ha emprendido por encargo de mediocres. El Espectador, Bogotá, 28 mar. 2018.

CÁRDENAS BARRETO, J. L. Conflictos de la filosofía experimental en el siglo XVII. Praxis Filosófica, Cali, n. 41, p. 57-80, 2015.

CUPANI, A. La peculiaridad del conocimiento tecnológico. Scientiæ Studi, São Paulo, v. 4, n. 3, p. 356-371, 2006.

DÍAZ CABALLERO, J. R. Humanismo y tecnología. Revista Cubana de Ingeniería, La Habana, v. 1, n. 2, p. 61-64, 2010.

Educação \& Formação, Fortaleza, v. 4, n. 12, p. 3-20 set./out. 2019

DOI: https://doi.org/10.25053/redufor.v4i12.1425

http://seer.uece.br/redufor 
DíAZ CABALLERO, J. R. La ciencia nacional. Revista Cubana de Ingeniería, La Habana, v. 7, n. 1, p. $54-62,2016$.

DÍAZ CABALLERO, J. R. (Coord.). Tecnología y sociedad. La Habana: Félix Varela, 2006.

DÍAZ CABALLERO, J. R.; BORRERO, I. S. Hacia dónde va la tecnología? Editorial Científico-Técnico, La Habana, 2011.

DíAZ CABALLERO, J. R.; RUBIDO RODRíGUEZ, E. Tecnología, sociedad y futuro. Revista Cubana de Ingeniería, La Habana, v. 1, n. 2, p. 61-68, 2011.

FEENBERG, A. O que é filosofia da tecnologia? In: NEDER, R. (Org.). A teoria crítica de Andrew Feenberg: racionalização democrática, poder e tecnologia. Brasília, DF: UnB, 2010. p. 39-51.

GATTI, B. A. Estudos quantitativos em educação. Educação e Pesquisa, São Paulo, v. 30 , n. 1, p. 11-30, 2004.

GONZÁLEZ GARCíA, M.; LÓPEZ CEREZO, J. A.; LUJÁN, J. L. Ciencia, tecnología y sociedad: una introducción al estudio social de la ciencia y la tecnología. Tecnos, Madrid, 1996.

GUTIÉRREZ, M. V. H.; ACHÓN, T. H. La formación de ingenieros desde el enfoque Ciencia, Tecnología y Sociedad. Referencia Pedagógica, La Habana, n. 1, p. 16-28, 2018.

ISNAD, G. D. The philosophy of technology and engineering. Journal of University Research, [S.I.], v. 1, n. 1, p. 7-13, 2018.

$\mathrm{KOERICH}, \mathrm{M}$. S. et al. Tecnologias de cuidado em Saúde e Enfermagem e suas perspectivas filosóficas. Texto Contexto - Enfermagem, Florianópolis, v. 15, n. esp., p. 178-185, 2006.

KUHN, T. S. A estrutura das revoluções científicas. São Paulo: Perspectiva, 1997.

LAMAR, A. R.; ROACH, E. F. F. La concepción de la ciencia y la técnica de Juan David García Bacca. In: SEMINARIO INTERNACIONAL SOBRE PENSAMIENTO LATINOAMERICANO, 1., 1987, La Habana. Resúmenes del Seminario. La Habana: Universidad de la Habana, 1987.

MEDINA, M. Tecnología y filosofía: más allá de los prejuicios epistemológicos y humanistas. Isegoria, [S.I.], v. 12, p. 180-197, 1995.

MITCHAM, C. La importancia de la filosofía para la ingeniería. In: LÓPEZ CEREZO, J. A. et al. (Ed.). Filosofía de la Tecnología. Madrid: Teorema, 2001. p. 31-53.

MITCHAM, C. ¿Qué es la Filosofía de la Tecnología? Barcelona: Anthropos, 1989. 
MONTERO CABRERA, L. La ciencia y la tecnología en Cuba una década después. Temas, n. 93-94, p. 4-10, 2018.

MORAN SEMINARIO, H. M. Filosofía de la Tecnología, su devenir y tendencia fundamentales. Revista de la Facultad de Ciencias Económicas, Lima, v. 11, n. 29, p. 117-130, 2006.

NÚÑEZ JOVER, J. La ciencia y la tecnología como procesos sociales. La Habana: Félix Varela, 2001.

OLIVEIRA, E. R. A. A filosofia da ciência e a sua extensão à engenharia. Millenium: Revista do Instituto Superior Técnico, Viseu, n. 24, p. 101-124, 2001.

\section{Adolfo Ramos Lamar (Brasil, Santa Catarina, Blumenau)} Universidade Regional de Blumenau (FURB)

Postdoctor en Educación por la Universidad de São Paulo (USP), doctor en Educación por la Universidad Estatal de Campinas (Unicamp) y mestre en Política Científica y Tecnología también por la Unicamp. Profesor en FURB.

Lattes: <http://lattes.cnpq.br/2295885579063119>.

E-mail: <ajemabra@yahoo.com.br>.

\section{Eduardo Francisco Freyre Roach (Cuba, Mayabeque, San José de las Lajas)} Universidad Agraria de la Habana (UNAH)

Postdoctor en Educación por la Universidad Estatal de Campinas (Unicamp) y doctor en Filosofía por la Universidad Estatal de Moscú, Rusia. Fue profesor titular en UNAH y profesor de maestría en Enseñanza Superior, Agroecología y Extensión Agrícola y de doctorados en Desarrollo Rural y Agrario. Ha impartido cursos en universidades de Brasil, México, Ecuador y Bolivia.

Lattes: <http://lattes.cnpq.br/9949395783481799>.

E-mail: <freyre.roach2016@gmail.com>.

Recibido el 4 de junio de 2019.

Aceptado el 2 de agosto de 2019. 